\title{
NUMERICAL INVESTIGATION, INCLUDING EXPERIMENTAL VALIDATION, OF AN AXIALLY BLOWN, STABLE ARC IN ARGON
}

\author{
B. Galletti ${ }^{a, *}$, F. Kassubek ${ }^{a}$, M. Buffoni $^{a}$, J. Carstensen $^{a}$, \\ P. C. StOlleR ${ }^{b}$ \\ ${ }^{a}$ ABB Switzerland Ltd., Corporate Research, 5405 Baden-Daettwil, Switzerland \\ ${ }^{b}$ ABB Switzerland Ltd., Technology Center - Interrupter Development, 5401 Zurich, Switzerland \\ * bernardo.galletti@ch.abb.com
}

\begin{abstract}
In this work we present the outcome of a numerical validation study conducted with an arc model developed within a computational fluid dynamics (CFD) tool. The numerical investigations were aimed at reproducing the spatially resolved temperature data obtained with an experiment in which an axially symmetric argon arc was established in the observation region. The full absorption spectrum has been computed for argon and then compressed with minimum loss of information to a relatively small set of bands. The latter has been used for solving the radiative transfer equation in a computationally affordable, yet accurate way. The comparison between the arc temperature simulated with the reduced absorption data and the measured one is presented.
\end{abstract}

Keywords: high-voltage circuit breaker, radiation, optical diagnostics, arc simulation, validation.

\section{Introduction}

The behavior of the electric arc in high voltage gas circuit breakers is the result of the balance between the Joule heating due to the current flowing through it and several mechanisms of removal and redistribution of the energy, such as radiation, convection and turbulent mixing. Moreover, the arc interacts with the solid surfaces around it, generating ablation from the insulating nozzles and erosion from the contacts. As a result, the gas that enters in the arc has a multi-species composition, whose complexity is further enhanced by the dissociation and ionization processes caused by the strong ohmic power density. Developing a high-fidelity numerical description of these arc phenomena is challenging, not only because of their inherent complexity, but also because of the strong coupling between them. For this reason, we have conceived an experiment [1] for measuring the spatially resolved temperature of an electric arc in a part of which some of the physical aspects described above can be disregarded, thus allowing a better validation of the physical and numerical models of the remaining ones. In particular, since in this experiment the arc was blown along two opposite axial directions, the effects of ablation, erosion and turbulence were minimized in the measurement window, located around the stagnation point. Another important feature of the test setup was that it achieved the stabilization of a cylindrical arc column in the observation region, thanks to the combined effects of bi-directional axial blowing and expansion of the gas from rest. The latter condition was obtained by pressurizing the test setup, so that the arc was drawn in high-pressure argon that discharges to ambient with small flow velocities upstream from the arc. The spatially resolved arc temperature was obtained through the application of non-intrusive diagnostics techniques based on imaging interferometry [1]. The arc stability achieved in this experiment not only enhanced significantly the measurements accuracy and their repeatability, but brought benefits also on the numerical side. It has, in fact, enabled the use of two-dimensional axisymmetric models, which allows a higher numerical accuracy for the same computational cost as a full three-dimensional model. In view of the above, the main physical aspects that we have tried to represent numerically were the correct characterization of the gas properties and the radiation transport; the latter being the topic on which the present work is focused. The thermodynamics and transport properties of the gas in the conditions existing in a plasma arc have been investigated by many authors, either assuming local thermodynamic equilibrium or departures from these equilibrium conditions, e.g. [2]. The available studies concerning radiation are focused on both the calculation of the full absorption spectra and on simplified models that reduce the complexity of the problem to a level that make them suitable for engineering applications [3]. The physical model of the arc in the experiments used for the numerical validation is discussed in Section 2, while Section 3 presents the numerical methodology and the comparison between simulation and experimental results.

\section{The physical model}

The experimental reference for the numerical validation campaign was obtained with the test device sketched in Figure 1 of [1]. Our arc simulation tool can take into account nozzle ablation and contact erosion. However, they were excluded from present numerical investigation, since we have verified that 
they did not influence the arc behavior in the nearstagnation region, where the physical model is to be validated. In fact, the material generated by erosion and ablation of the surfaces exposed to the arc is conveyed downstream by the strong bidirectional axial flow. However, the ablation has an indirect impact on the flow at stagnation as it is responsible for a slight widening of the nozzle throat.

The physical model that describes the behavior of the plasma arc in this experiment has been derived under the assumption that the conditions for the chemical equilibrium (CE) and local thermodynamic equilibrium (LTE) are fulfilled. The governing equations of the arc model used in this work are:

$\partial_{t} \rho+\nabla \cdot(\rho \mathbf{U})=0$

$\rho D_{t} \mathbf{U}=-\nabla p+\nabla \cdot \boldsymbol{\tau}-\sigma \nabla \varphi \times(\nabla \times \mathbf{A})$

$\rho D_{t} h^{o}=\partial_{t} p+\nabla \cdot(k \nabla T+\boldsymbol{\tau} \cdot \mathbf{U})-\nabla \cdot \mathbf{q}_{\mathrm{rad}}+\sigma(\nabla \varphi)^{2}$

$\nabla \cdot(\sigma \nabla \varphi)=0$

$\nabla^{2} \mathbf{A}=\mu_{0} \sigma \nabla \varphi$

$\hat{\mathbf{s}} \cdot \nabla I_{\lambda}=a_{\lambda}\left(I_{b \lambda}-I_{\lambda}\right)$

where $D_{t}=\partial_{t}+\mathbf{U} \cdot \nabla$ denotes the material derivative.

Equations (1), (2) and (3) express the conservation of mass, momentum, and energy of the gas, respectively. In these equations, $\rho$ is the mass density, $\mathbf{U}$ is the velocity vector, $p$ is the pressure, $\boldsymbol{\tau}$ is the stress tensor, $h^{o}$ is the total enthalpy per unit mass, $k$ is the thermal conductivity, $T$ is the temperature, $\mathbf{q}_{\mathrm{rad}}$ is the radiative heat flux, $\varphi$ is the electric potential, A is the magnetic vector potential, $\sigma$ is the electrical conductivity, and $\mu_{0}$ is the magnetic constant.

Equations (4) and (5) represent current conservation and Ampere's law in the Coulomb gauge, respectively, under the magnetostatics approximation. The latter is valid when, as in the present case, the derivatives of electromagnetic fields can be neglected. The Lorentz force per unit volume $-\sigma \nabla \varphi \times(\nabla \times \mathbf{A})$ was numerically proven to have a negligible influence on the arc, which was expected as the arc was strongly axially blown and the currents were of the order of $100 \mathrm{~A}$.

The model is completed by the quasi-steady radiative transfer equation (RTE) (6) for a non-scattering medium with absorption coefficient $a_{\lambda}$. The RTE governs the transport through the gas of the spectral radiative intensity $I_{\lambda}(\mathbf{r}, \hat{\mathbf{s}})$, which is the amount of radiative power in the wavelength interval $[\lambda, \lambda+d \lambda]$ at position $\mathbf{r}$ in the direction $\hat{\mathbf{s}}$ per unit solid angle and unit area normal to $\hat{\mathbf{s}}[4]$. The term $I_{b \lambda}(\mathbf{r})$ indicates the spectral radiative intensity for a blackbody. The RTE is bidirectionally coupled with the energy equation (3) through the divergence of the radiative heat flux $\mathbf{q}_{\text {rad }}=\int_{0}^{\infty} \int_{4 \pi} I_{\lambda} \hat{\mathbf{s}} \mathrm{d} \Omega \mathrm{d} \lambda$ and through the absorption coefficient $a_{\lambda}(\mathbf{r})$, which in local thermodynamic equilibrium is a function only of pressure and temperature of the gas at the position $\mathbf{r}$.

With regard to turbulence, an axially blown arc induces turbulence through the flow in which it passes.
The reason is the copresence of a radial gradient of the density across the arc with an axial gradient of the pressure due to the flow expansion. These two gradients result in a strong baroclinic production term of vorticity, $S_{\omega}=\nabla p \times \nabla \rho^{-1}$, which has its largest magnitude inside the arc mantle where $\nabla \rho$ is maximal, and is equal to zero at the stagnation point where $\nabla p$ is zero [5]. The arc can be then thought of as having a nearly irrotational core surrounded by a turbulent mixing zone. Since in our experiment the flow reaches the stagnation point almost undisturbed and $S_{\omega}=0$ at stagnation, the flow can be considered laminar in the near-stagnation region where the arc model validation is performed. The turbulent mixing becomes more and more significant as the flow moves downstream along the two opposite axial directions. However, the throats of both the upper electrode and the nozzle are choked during the tests. Thus, the flow conditions around the stagnation point are unaffected by the downstream turbulent mixing. For these reasons, we have used the laminar flow model in our simulations. In fact, the validity of this approach has been confirmed by comparing the laminar flow quantities around stagnation with those computed with the $k \omega$-SST turbulence model. The differences were quite small if the Kato-Launder correction [6] was applied for suppressing the spurious generation of turbulent kinetic energy in the stagnation region.

The thermodynamics and transport properties of argon have been computed by an in-house Cantera based tool [7] that includes Debye-Hückel non-ideality corrections under the $\mathrm{CE}$ and LTE assumptions. These properties, functions of temperature and pressure, were stored as lookup tables to be used within the CFD solver. With the same in-house we have computed the absorption spectrum of argon using the databases [8, 9].

\section{The numerical model}

The simulations were performed with the computational domain depicted in Figure 1 along with the pressure sensors' locations, in the tank $\left(S_{T}\right)$ and along the nozzle $\left(S_{1}, S_{2}, S_{3}\right)$.

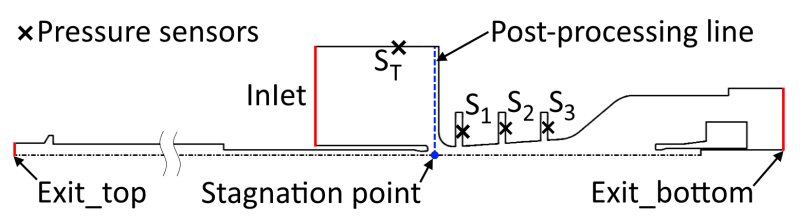

Figure 1. 2D-axisymmetric computational domain with plug in full-open position.

This is a two-dimensional axisymmetric domain which is derived with some modifications from the test device geometry used in [1]. In particular, the tank was not modeled in its entirety, in that its top boundary was shifted close to the nozzle and converted from wall into an inlet at which the measured pressure was prescribed. This sped up the simulations without 
affecting the accuracy, as we have verified by comparing the pressure distribution along the nozzle with that of the case in which the full tank discharge was simulated. At the bottom outlet the ambient pressure was prescribed, while at the top outlet we imposed the time-decaying pressure read from a sensor placed at the end of the hollow contact and immediately upstream from the system of hoses that redirect the flow to the bottom part of the pressurized volume [1]. The pressure was measured at three axial locations along the nozzle.

The physical model described above was numerically implemented in ANSYS Fluent 17.1. This commercial software solves for the flow equations with the finite-volume discretization method. It offers the user the freedom to implement a generic transport equation for the scalar $\phi$, of the following form $\partial_{t}(\rho \phi)+\nabla \cdot(\rho \mathbf{U} \phi)=\nabla \cdot(\Gamma \nabla \phi)+S_{\phi}$. The steady version of this transport equation with no convection term can be used for solving equations (4) and (5), by properly selecting the diffusivity coefficient $\Gamma$ and the the source term $S_{\phi}$. A structured mesh was used everywhere in the computational domain. The spatial resolution and the time stepping were selected in order to ensure a properly converged solution. The grid was refined, and the time steps reduced until a satisfactory grid convergence was achieved. The motion of the plug that draws the arc between the two hollow contacts was simulated as a moving porous region impermeable to the gas and having the same electrical and thermal conductivities as the plug. This numerical strategy allowed for no mesh alterations, preserving its high quality during the contact opening phase. The numerical integration of the RTE (6) in ANSYS Fluent was done with the gray-band discrete ordinates (GBDO) radiation model, due to the fine spectral and spatial resolution it allows. In this model, the RTE is solved for a finite number of discrete solid angles, each associated with a direction vector $\hat{\mathbf{s}}_{j}$, and for a finite number of bands $\left[\lambda_{i}, \lambda_{i+1}\right]$ in which the absorption spectrum is divided, with the assumption that the absorption coefficient is constant within each band. Under these conditions and assuming a refractive index of 1 for argon, the (6) and the divergence of the radiative heat flux can be written as:

$$
\begin{aligned}
& \pi\left(\hat{\mathbf{s}}_{j} \cdot \nabla \mathcal{J}_{i, j}+a_{i} \mathcal{J}_{i, j}\right)=\sigma_{\mathrm{sb}} T^{4} a_{i} \mathcal{E}_{i} \\
& \nabla \cdot \mathbf{q}_{\mathrm{rad}}=\sum_{i=1}^{N_{\mathrm{bands}}}\left[4 \sigma_{\mathrm{sb}} T^{4} a_{i} \mathcal{E}_{i}-a_{i} \sum_{j=1}^{N_{\mathrm{beams}}} \mathcal{J}_{i, j} \Delta \Omega_{j}\right]
\end{aligned}
$$

which are the equations of the GBDO model. In (7), $i$ runs from 1 to $N_{\text {bands }}$ and $j$ from 1 to $N_{\text {beams. For }}$ the remaining notations, $I_{i, j}$ is the radiative intensity for the i-th band $\left[\lambda_{i}, \lambda_{i+1}\right]$ and in the beam of solid angle $\Delta \Omega_{j}$ along the $\hat{\mathbf{s}}_{j}$ direction, $\mathcal{J}_{i, j}=\int_{\lambda_{i}}^{\lambda_{i+1}} I_{i, j} d \lambda=$ $I_{i, j}\left(\lambda_{i+1}-\lambda_{i}\right)$ is the band-integrated radiative intensity for the j-th beam, $a_{i}$ is the absorption coefficient in the i-th band, $\sigma_{\mathrm{sb}}$ is the Stefan-Boltzmann constant,
$\mathcal{E}_{i}=f\left(\lambda_{i+1} T\right)-f\left(\lambda_{i} T\right)$ is the emissivity weighting factor, i.e. the fraction of the total energy emitted by a black body in the i-th band at the temperature $T$. The integral function $f(\lambda T)$ represents the fraction of the same energy in the wavelength interval from 0 to $\lambda$ and can be found tabulated (e.g. in [4]). In case of rotational symmetry, Fluent discretizes only half of the full angular space, by dividing it in four octants, each of which is in turn divided into $N_{\theta} \times N_{\phi}$ solid angles. We have retained the default division $N_{\phi}=$ $N_{\theta}=2$, which yields a total of $16 N_{\text {bands }}$ equations of type (7) that are to be solved at each cell of the computational domain. The linearity of the equations (7) and (8) with respect to the product $a_{i} \mathcal{E}_{i}$ implies that the solution of the radiation problem does not change if two or more bands (e.g. bands $\left[\lambda_{p}, \lambda_{p+1}\right]$ and $\left.\left[\lambda_{q}, \lambda_{q+1}\right]\right)$ in which the absorption coefficient value is the same (e.g. $a_{p}=a_{q}$ ) are grouped together, and if the new grouped band is given the emissivity weighting factor that is the sum of those of the original bands (e.g. $\varepsilon_{p}+\varepsilon_{q}$ ). ANSYS Fluent provides a macro for assigning directly the emissivity weighting factors of the bands, which makes it straightforward to use the band grouping technique described above. It should be noted that in this case the solution no longer depends on the wavelength limits of the grouped bands, as these are already included in the $\mathcal{E}_{i}$ jumps. This method was generalized to the full argon spectrum which contains about $10^{6}$ wavelengths, see Figure 2.

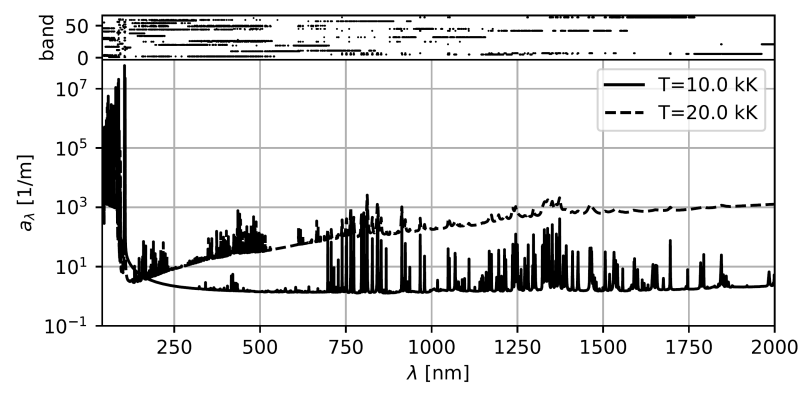

Figure 2. Argon absorption spectrum for different temperatures at 10 bar. Top graph: attribution of the wavelength range to the different bands in the 64 band model.

The idea was to group together those wavelengths in which the absorption coefficient had values comprised in a certain small range. However, a partition of a single spectrum in segments with similar $a_{\lambda}$ is unfortunately not sufficient; as seen in Figure 2, absorption spectra differ strongly depending on temperature. This is due to the different composition of the plasma at different temperatures; at low temperatures atomic lines dominate whereas at higher temperatures ion lines appear. We have implemented an algorithm that, for a given pressure, groups together bands $\left[\lambda_{i}, \lambda_{i+1}\right]$ whose $a_{i}(T)$ coefficients differ from each other only slightly over the full temperature range. The distance between these $a_{i}(T)$ is determined based on an appropriate functional norm. The details of this procedure will be illustrated in a separate article. The points in 
the upper part of Figure 2 show the results of this technique for the compression of the full spectrum into 64 grouped bands. A detailed analysis shows that indeed, spectral lines of the same origin (i.e. same species and spectroscopic term) are typically found in the same clustered band. The validation of the absorption data obtained with this procedure was accomplished by solving the RTE for a rotationally symmetric distribution of temperature $T(x, r, \theta)=T_{\exp }(r)$, where $T_{\exp }(r)$ was the radial distribution measured at stagnation at $p=12.7$ bar in [1]. In Figure 3, $T_{\exp }(r)$ is depicted along with the comparison between the $\mathbf{q}_{\mathrm{rad}}$ computed by integrating the RTE with a 64-band grouped absorption spectrum and with the full argon spectrum. In the latter case, the RTE was solved at 10 radial positions only.

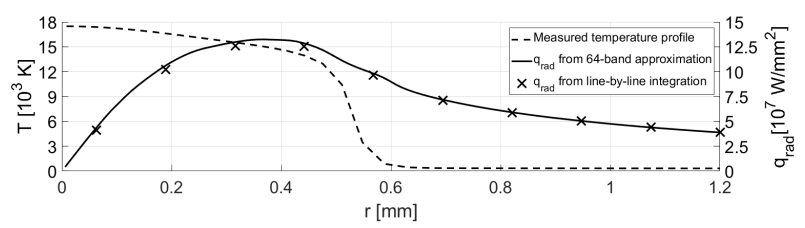

Figure 3. Radiative heat flux computed with the full spectrum and with its 64-band approximation

As it can be seen, the reduced spectrum with 64 grouped bands gives an accurate representation of the radiation transport with a maximum relative error on $\mathbf{q}_{\mathrm{rad}}$ of $6 \%$ with respect to the full spectrum.

\subsection{The results of the validation}

The 64-band reduced absorption data was used in the numerical reproduction of the spatially resolved measurements of the arc temperature obtained in [1]. That experimental campaign consisted of several arcing operations (shots), each of which resulted in a slight change of the nozzle contour due to arc-induced ablation. The nozzle in the shot that we have selected for the numerical reproduction (shot \#41) was not new, thus we increased its throat diameter of $0.5 \mathrm{~mm}$, so that the simulated pressure matched the values measured at $S_{1}, S_{2}, S_{3}$. In this test a slowly decaying quasi-DC current was injected through the system that had a value of $158 \mathrm{~A}$ at the instant in which the spatially resolved measurements of the arc were acquired at stagnation. At the same instant the pressure in the tank pressure sensor reading was 11.8 bar, against an initial value of 12.8 bar. In Figure 4 the measured and simulated temperature profiles are represented along the radial post-processing line depicted in Figure 1.

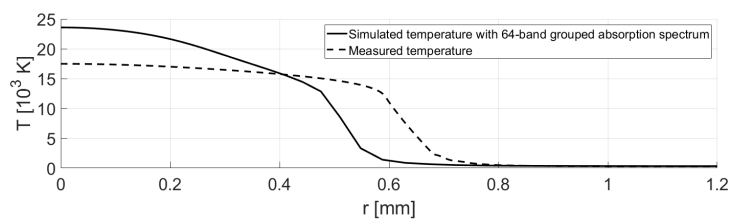

Figure 4. Simulated vs. measured radial profile of the temperature at stagnation.
Both profiles refer to the same axial position close to the stagnation point and to the same time. As can be seen, the numerical model underpredicts by $17 \%$ the measured arc radius. The predicted arc temperature at the axis is instead overestimated by $25 \%$.

\section{Conclusions}

In this work, we have presented the results of the validation study conducted for a CFD-based arc model and aimed at reproducing the spatially resolved temperature measurements of a stabilized axially-blown arc. The test device was conceived so that ablation, erosion and turbulence could be neglected in the observation region, around its stagnation point, thus allowing a more precise validation of the radiation model, which was the main focus of this article. The full absorption spectrum has been computed for argon and then compressed to 64 clustered bands that were proven to approximate it very well. This reduced absorption spectrum was then used as an input for the gray-band discrete ordinates model of ANSYS Fluent to perform arc simulations in an accurate and computationally affordable way. Despite the high precision with which the radiation transport has been resolved numerically, the simulated arc temperature distribution exhibited a non-satisfactory agreement with the measured one. An additional research effort is thus needed on both the experimental and numerical side to further refine the diagnostics technique and to assess the effect of the implementation of non-equilibrium phenomena on the numerical representation of the arc behavior.

\section{References}

[1] J. Carstensen et al. Measuring density profiles of electrons and heavy particles in a stable axially blown arc. Phys. Rev. Appl., 8(2):024002, 2017. doi:10.1103/PhysRevApplied.8.024002.

[2] A. Gleizes et al. Topical review: Thermal plasma modelling. J. Phys. D: Appl. Phys., 38:R153-R183, 2005. doi:10.1088/0022-3727/38/9/R01.

[3] L. Fagiano and R. Gati. On the order reduction of the radiative heat transfer model for the simulation of plasma arcs in switchgear devices. JQSRT, 169:58-78, 2016. doi:10.1016/j.jqsrt.2015.10.002.

[4] M. F. Modest. Radiative Heat Transfer (Third Edition). Academic Press, 2013.

[5] L. Niemeyer et al. On the development of turbulence by the interaction of gas flows with plasmas. In AIAA 9th Electr. Propul. Conf., pages 1-9, Bethesda, April 1972.

[6] M. Kato and B. E. Launder. The modelling of turbulent flow around stationary and vibrating square cylinders. In Proc. 9th Symp. on Turbulent Shear Flows, pages 10.4.1-10.4.6, Kyoto, August 1993.

[7] C. Doiron and K. Hencken. APS Meeting Abstracts, 3:3001, 2013.

[8] Kurucz database. http://kurucz.harvard.edu/.

[9] TopBase database. http:

//cdsweb.u-strasbg.fr/topbase/topbase.html. 